\title{
Effects of Different Doses of Stereotactic Radiotherapy on Recurrence, Metastasis and Survival of Patients with Lung Cancer
}

\author{
Liwei Zhang \\ Hefei Cancer Hospital, Chinese Academy of Sciences, Hefei 230031, Anhui Province, China
}

\begin{abstract}
Objective: To investigate the effect of different doses of stereotactic radiotherapy (SBRT) on the recurrence, metastasis and survival of lung cancer patients. Methods: The clinical data of 13 patients with lung cancer who were treated in our hospital from May 2016 to June 2020 and were followed up for one year were retrospectively analyzed. The patients treated with low-dose SBRT were divided into the observation group (7 cases) and the patients treated with high-dose SBRT were divided into the control group (6 cases). The clinical efficacy, recurrence, metastasis, survival status and incidence of adverse reactions were compared between the two groups. Results: There were no significant differences in the clinical total effective rate, 1-year recurrence rate, metastasis rate and survival rate between the two groups $(P>0.05)$; The incidence of adverse reactions in the observation group was lower than that in the control group, the difference was statistically significant $(P<0.05)$. Conclusion: High dose or low dose SBRT can achieve good curative effect and prognosis in patients with lung cancer, but low dose SBRT has less adverse reactions and higher safety.
\end{abstract}

Key words: Lung cancer; Stereotactic radiotherapy; Recurrence; Transfer; Living conditions

Publication date: March, 2021

Publication online: 31 March, 2021

"Corresponding author: Liwei Zhang, doctorzhang liwei@163.com

Lung cancer is a common clinical malignant tumor of the lung. Its clinical manifestations include blood in sputum, cough and chest pain. Because its early symptoms are not significant, the disease is often diagnosed in the middle and late stage, which has a high clinical mortality. At present, radiotherapy, chemotherapy, surgery and targeted therapy are often used in clinic. Stereotactic radiotherapy (SBRT) is an innovation on the basis of traditional radiotherapy. By increasing the dose of each radiotherapy, it can reduce the number of times of radiotherapy, enhance the radiation concentration of the focus area, and reduce the damage to tissues outside the focus.However, at present, there is no relevant standard for the dosage of SBRT, and there are few special reports on this kind of research in academic circles ${ }^{[1]}$. In view of this, the purpose of this study was to investigate the effect of different doses of SBRT on recurrence, metastasis and survival of lung cancer patients. It is shown as follows.

\section{Material and methods}

\subsection{General information}

Clinical data of 13 patients with lung cancer admitted to our hospital from May 2016 to June 2020 and were followed up for one year were retrospectively analyzed. Patients treated with low-dose SBRT were classified as the observation group ( 7 cases), and patients treated with high-dose SBRT were classified as the control group (6 cases). There were 5 males and 2 females in the observation group; The average age was $(61.87 \pm 1.26)$ years old; The tumor diameter was $2-6.5 \mathrm{~cm}$, with an average of $(4.18 \pm 0.53) \mathrm{cm}$; Histological type: There were 2 cases of adenocarcinoma, 3 cases of squamous cell carcinoma, and the other 2 cases; Tumor (TNM) 
stage: 1There were 1 case of stage I, 3 cases of stage II and 3 cases of stage III a ${ }^{[2]}$. There were 4 males and 2 females in the control group; The average age was $(61.93 \pm 1.18)$ years (range, $52-71$ years); The tumor diameter was $2.5-7 \mathrm{~cm}$, with an average of (4.27 $\pm 0.49) \mathrm{cm}$; Histological type: There were 2 cases of adenocarcinoma, 3 cases of squamous cell carcinoma and 1 case of the rest; TNM stage: There were 1 case in stage I , 3 cases in stage $I$ and 2 cases in stage III a. There was no significant difference between the two groups $(P>0.05)$.

\subsection{Diagnostic criteria}

All patients were in accordance with the diagnostic criteria of lung cancer in the code for diagnosis and treatment of primary lung cancer. (1) The clinical manifestations were as follows: Irritative dry cough, blood in sputum or blood sputum, chest pain, fever or shortness of breath, the above symptoms were more than 14 days and could not be relieved after treatment; (2) Physical examination: Extrapulmonary signs with unknown causes and long-term treatment were accompanied by hepatomegaly, subcutaneous nodules and vocal cord paralysis; (3) Imaging examination: Chest X-ray examination; Chest CT examination; B-mode ultrasonography; MRI examination; Fiberoptic bronchoscopy; Mediastinoscopy.

\subsection{Inclusion criteria}

(1) Inclusion criteria: All of them met the above diagnostic criteria; Lung cancer was diagnosed by pathology; The clinical data were complete.

(2) Exclusion criteria: Patients with secondary lung cancer; Contraindications of CyberKnife; Patients with distant metastasis; Patients with previous chemoradiotherapy history; Patients with abnormal blood system; Patients with immune dysfunction ${ }^{[3]}$.

\subsection{Methods}

Two groups of patients were given routine treatment of lung cancer, guiding patients to pay attention to rest, diet should follow the principle of high protein, low $\mathrm{fat}^{[3]}$. Before treatment, all patients underwent routine examination of blood routine, urine routine, liver function and chest $\mathrm{CT}$.

\subsubsection{The observation group was treated with low- dose SBRT}

During the treatment, the patient was placed in supine position and asked to breathe gently. After the patient was fixed with positioning frame, Philips brilliance CT big produced by Philips electric medical systems was used. The target area was determined by CT simulation positioning. The scanning slice thickness was $1.25 \mathrm{~mm}$, and the scanning range was $15 \mathrm{~cm}$ above and below the lesion. After uploading the acquired images to the workstation, the target area and organs at risk were outlined according to the images. The lesions were irradiated by $6 \mathrm{D}$ axesse high-energy linear accelerator produced by electa according to the CT images5-8gy/time, 5 times/ week or 3 times/week, every other day treatment, generally a total of about 7-15 times of irradiation, the biological effect dose (bed) is about 70-105gy ${ }^{[4]}$.

\subsubsection{The control group was treated with high-dose SBRT}

The control group was treated with high-dose SBRT and the CT simulation positioning steps were the same as those of the observation group5-6gy / time, 5 times / week, a total of about 12 times, the bed is about 90-105gy. The two groups were followed up for 1 year after discharge.

\subsection{Observation indexes}

The clinical efficacy, recurrence, metastasis, survival status and the incidence of adverse reactions in 2 groups were compared 1 year after discharge. (1) The clinical efficacy was evaluated according to the evaluation criteria of solid tumor in the code for diagnosis and treatment of primary lung cancer. The tumor disappeared completely for more than one month; Partial remission (PR): The tumor volume decreased by more than $50 \%$ and lasted for more than 1 month; Stable (SD): The tumor volume increased by less than $25 \%$ and lasted for more than 1 month; Progress (PD): The tumor volume increased by more than $25 \%$; Total effective rate $=(\mathrm{Cr}+\mathrm{PR})$ cases $/$ total cases $\times 100 \%$. (2) Adverse reactions included radiation pneumonitis, cough, nausea and vomiting, and atelectasis.

\subsection{Statistical methods}

SPSS 20.0 software was used to analyze the data, to express the measurement data. Independent sample t-test was used between the groups. The count data was expressed by percentage. $\chi^{2}$ was used. Fisher exact test was used for small sample data. $P<0.05$ was considered as statistically significant. 


\section{Results}

\subsection{Clinical efficacy}

After the treatment, there were 4 cases of CR, 1 case of PR, 2 cases of SD and 0 cases of PD in the observation group. The total effective rate was $71.43 \%$ in 5 cases. There were 3 cases of $\mathrm{Cr}, 1$ case of PR, 2 cases of SD and 0 case of PD in the control group. The total effective rate was $66.67 \%$. There was no significant difference in the total effective rate between the two groups $\left(\chi^{2}=0.000, P=1.000\right)$.

\subsection{Statistics of serum PTEN and SAA levels}

According to the statistics in the table below, there was no significant difference in serum PTEN and SAA levels between the two groups before and after treatment, $P>0.05$.

Table 1. Statistical Evaluation of two groups of indicators

\begin{tabular}{cccccc}
\hline \multirow{2}{*}{ Group } & \multicolumn{2}{c}{ PTEN } & & \multicolumn{2}{c}{ SAA } \\
\cline { 2 - 3 } \cline { 5 - 6 } & Before treatment & After treatment & & Before treatment & After treatment \\
\hline Control group $(n=6)$ & $0.38 \pm 0.04$ & $0.81 \pm 0.15$ & $406.38 \pm 29.54$ & $144.25 \pm 11.38$ \\
Observation group $(n=7)$ & $0.36 \pm 0.05$ & $0.76 \pm 0.20$ & $407.54 \pm 30.11$ & $146.53 \pm 13.66$ \\
$t$ & 0.7861 & 0.7456 & 0.0698 & 0.3233 \\
$P$ & 0.4484 & 0.4715 & 0.9456 & 0.7525 \\
\hline
\end{tabular}

\subsection{Recurrence, metastasis and survival status}

One year after discharge, there was no recurrence of lung cancer in the observation group. Metastasis occurred in 1 case $(14.29 \%) ; 6$ cases survived and 1 case died. The survival rate was $85.71 \%$; In the control group, lung cancer recurred in 1 case, with a recurrence rate of $16.67 \%$; No metastasis occurred; The survival rate was $83.33 \%$. There were no significant differences in 1-year recurrence rate, metastasis rate and survival rate between the two groups $\left(\chi^{2}=0.006,0.000,0.000 ; \chi^{2}=0.000,0.000 ; \chi^{2}=\right.$
$0.000) ; P=0.936$ 、1.000、1.000).

\subsection{Adverse reactions}

As can be seen from the following table, no adverse reactions occurred in the observation group, with an incidence of $0 \%$. In the control group, there were 2 cases of radiation pneumonia, 1 case of cough, 1 case of nausea and vomiting, and the incidence rate was $66.67 \%$; The incidence of adverse reactions in the observation group was lower than that in the control group, and the difference was statistically significant $\left(\chi^{2}=3.974 ; P=0.046\right)$.

Table 2. Statistics of adverse reactions

\begin{tabular}{ccccc}
\hline Group & cough & Nausea and vomiting & Radiation pneumonitis & Total incidence \\
\hline Control group $(n=6)$ & 1 & 1 & 2 & 66.67 \\
Observation group (N7) & 0 & 0 & 0 & 0.00 \\
$\chi^{2}$ & & & 3.9740 \\
$P$ & & & 0.0460 \\
\hline
\end{tabular}

\section{Discussion}

The course of lung cancer is slow, leading to no obvious symptoms in the early stage, and most of them are in the middle and late stage when diagnosed, so lung cancer has a high mortality ${ }^{[5]}$. At present, the medical community believes that thorough removal of the focus is the main way to treat lung cancer, but some patients with advanced lung cancer are treated by surgery with a high recurrence rate, which makes some patients need to receive secondary treatment, increasing the pain and economic burden of patients ${ }^{[6]}$. Therefore, for patients with advanced lung cancer, radiotherapy and chemotherapy is still the main method. As a new radiotherapy method, SBRT can accurately control the target area and radiation dose, which has important clinical significance for improving the long-term survival rate of patients. However, there is no standard for the dose of SBRT.

The results showed that there was no difference in the total effective rate, 1-year recurrence rate, metastasis rate and survival rate between the two groups; The incidence of adverse reactions in the observation group was lower than that in the control group, indicating that high-dose or low-dose SBRT can achieve good curative effect and prognosis in lung cancer patients, but low-dose treatment has less adverse reactions and higher safety. The reason is that the main working principle of SBRT is to combine 
CT image guidance with respiratory management technology to obtain the CT images of patients when they breathe in and breathe out. The CT images can include the range of target area, organ movement and posture error of patients, and then guide the patients to adjust their breathing according to the location of lesions to achieve the effect of accurate positioning. Among them, CT image-guided technology can ensure the location of SBRT at the edge of the lesion, which has the advantages of small error and high accuracy. In this study, the positioning frame is used to fix the patient, which can reduce the error caused by the patient's movement. High dose irradiation can effectively kill tumor cells in the lesion area to control the progression of the lesion. However, the inactivation of tumor cells in high dose irradiation is relatively fast, and the continued irradiation after the inactivation of tumor cells may cause different degrees of damage to the healthy tissues around the lesion, which may cause many adverse reactions after treatment. Low dose irradiation can reduce the volume of lesions by reducing the radiation dose, reduce the damage to lung tissue and organs, and reduce the incidence of adverse reactions ${ }^{[7,8]}$. However, due to the small sample size and the short follow-up time after discharge, the conclusion of this study may be biased and not comprehensive. Therefore, it is necessary to further expand the sample size and extend the follow-up time to obtain more accurate and comprehensive research conclusions in the future.

In conclusion, patients with lung cancer using high-dose or low-dose SBRT can achieve good curative effect and prognosis, but low-dose treatment has less adverse reactions and higher safety.

\section{References}

[1] Ke LY, Liang DS, Song CL, et al. Effects of three kinds of radiotherapy on recent neurocognitive function and activities of daily living in patients with brain metastases from nonsmall cell lung cancer [J]. Journal of Guangdong Medical College, 2020, 38(1): 29-32.

[2] Yang Y, Su GH, Ma L. Efficacy evaluation and quality of life analysis of gyro knife stereotactic radiotherapy for early nonsmall cell lung cancer [J]Chinese Journal of Clinicians, 2020, 48(3): 334-336.

[3] Zou JH, Liu ZQ, Xu JF. Effect of 4D-CT on internal target volume and dose in stereotactic radiotherapy [J]. Chinese Journal of Medical Physics, 2020(8).

[4] Dai JY, Liu N, Xian P, et al. Clinical study of stereotactic radiotherapy combined with endocrine therapy for metastatic hormone sensitive prostate cancer [J]. Journal of Clinical Urology, 2020, V.35; No.319 (7): 6-10 + 16

[5] Yu HF, Yu ZL. Difference of curative effect between stereotactic radiotherapy and surgery for early non-small cell lung cancer $[\mathrm{J}]$. World Latest Medical Information Digest (electronic version), 2020, 20(1): 76-77.

[6] Chan S T, Ruan D, Shaverdian N, et al. Effect of radiation doses to the heart on survival for stereotactic ablative radiotherapy for early-stage non-small-cell lung cancer: An artificial neural network approach[J]. Clinical Lung Cancer, 2020, 21(2): 136-144.

[7] Coelho M, Squizzato A, Cassina, Niccolò, et al. Effect of betablockers on survival of lung cancer patients: a systematic review and meta-analysis[J]. European Journal of Cancer Prevention, 2020, 29.

[8] Wang XY, Cui CF, Wang MR, et al. The effect of stereotactic radiotherapy combined with cerebral vascular interventional embolization therapy on the neurologic function of hypervascular brain tumor $[\mathrm{J}]$. Chinese Journal of Clinical Oncology, 2020, 27(3): 53-56. 